\title{
Survival Analysis over 28 Years of 173,378 Patients with Hepatocellular Carcinoma in Japan
}

\author{
Masatoshi Kudo ${ }^{a}$ Namiki Izumi ${ }^{b}$ Michiie Sakamoto ${ }^{c}$ \\ Yutaka Matsuyama $^{d}$ Takafumi Ichida ${ }^{e}$ Osamu Nakashima ${ }^{f}$ \\ Osamu Matsuig Yonson Ku ${ }^{\mathrm{h}}$ Norihiro Kokudo \\ Masatoshi Makuuchij for the Liver Cancer Study Group of Japan
}

\begin{abstract}
aDepartment of Gastroenterology and Hepatology, Kindai University Faculty of Medicine, OsakaSayama, 'Department of Gastroenterology and Hepatology, Musashino Red Cross Hospital, Musashino, 'Department of Pathology, Keio University School of Medicine, Shinjuku, dDepartment of Biostatistics, School of Health Sciences and Nursing, University of Tokyo, Tokyo, eDepartment of Gastroenterology, Shizuoka Hospital Juntendo University School of Medicine, Shizuoka, fDepartment of Clinical Laboratory Medicine, Kurume University Hospital, Kurume, 9Department of Radiology, Kanazawa University Graduate School of Medical Science, Kanazawa, hDepartment of Surgery, Kobe University Graduate School of Medicine, Kobe, 'Department of Hepato-Biliary and Pancreatic Surgery, University of Tokyo Graduate School of Medicine, Tokyo, jDepartment of Surgery, Japanese Red Cross Medical Center, Tokyo, Japan
\end{abstract}

\section{Key Words}

Hepatic arterial infusion chemotherapy · Hepatocellular carcinoma ·

Liver Cancer Study Group of Japan · Nationwide survey · Overall survival

\begin{abstract}
Background: Beginning in 1967, the Liver Cancer Study Group of Japan (LCSGJ) started a nationwide prospective registry of all patients with hepatocellular carcinoma (HCC) diagnosed at more than 700 institutions. To determine the effectiveness of surveillance and treatment methods longitudinally, we analyzed improvements over time in overall survival (OS) of 173,378 patients with HCC prospectively entered into the LCSGJ registry between 1978 and 2005. Methods: All patients from more than 700 institutions throughout Japan with HCC were entered into the LCSGJ registry. Patients were grouped by years of diagnosis, with OS and 5-year OS rates being calculated. We also assessed OS and 5-year OS rates in patients who underwent resection, local ablation, transarterial chemoembolization (TACE), and hepatic arterial infusion chemotherapy (HAIC) and in those with baseline serum alpha-fetopro-
\end{abstract}


tein (AFP) levels $\geq 400 \mathrm{ng} / \mathrm{ml}$. Results: The 5- and 10-year OS rates in the cohort of 173,378 patients were $37.9 \%$ and $16.5 \%$, respectively. However, over time, the mean maximum tumor size decreased significantly, whereas 5-year OS rates and median survival time increased significantly. Similar findings were observed separately in patients who underwent resection, local ablation, TACE, and HAIC, as well as in patients with AFP levels $\geq 400 \mathrm{ng} / \mathrm{ml}$. Conclusion: The establishment of a nationwide HCC surveillance program in Japan has contributed to longer median OS and increased OS rates in patients diagnosed with this disease. These findings suggest that the establishment of a surveillance program in other countries with patients at risk for HCC may provide significant survival benefits.

Copyright (C) 2016 S. Karger AG, Basel

\section{Introduction}

Worldwide, hepatocellular carcinoma (HCC) is the sixth most common cancer and the third most common cause of cancer deaths [1]. Globally, about $80 \%$ of patients with HCC are in Asian countries [2, 3]. In Japan, the incidence of HCC and the associated death rates began to increase sharply in 1975 , peaking at 34,510 and $27.4 / 100,000$, respectively in 2004 . However, these decreased to 33,662 and $26.7 / 100,000$, respectively by 2006 [4]. The primary cause of HCC in Japan is infection with hepatitis C virus (HCV), which is observed in about $70 \%$ of all patients. This is followed by infection with hepatitis B virus in $15-22 \%$, and nonviral causes, which is predominantly alcohol associated, in the remaining $8-15 \%$ of patients. The survival of Japanese patients with HCC can be predicted by the Japan Integrated Staging score [5]. Risk factors for the development of HCC, methods of prevention, diagnosis and treatment modalities were recently reviewed [6].

Beginning in 1967, the Liver Cancer Study Group of Japan (LCSGJ) started a nationwide prospective registry of patients with HCC. Patients were followed-up after diagnosis with surveys published every two years [7, 8]. In the 18th and most recent follow-up survey for the period 2004-2005, 20,153 patients were newly diagnosed with HCC and 30,677 previously diagnosed patients were being followed-up at 544 institutions [8], respectively. This follow-up survey showed that, of 17,804 measurable tumors in patients initially diagnosed in the period 2004-2005, 855 (4.80\%) were $\leq 1 \mathrm{~cm}, 5,106$ (28.68\%) were $1-2 \mathrm{~cm}, 4,272$ (23.99\%) were $2-3 \mathrm{~cm}, 3,833$ (21.53\%) were $3-5 \mathrm{~cm}$, and 3,738 (21.00\%) were 5-10 cm in diameter, respectively. This survey also showed that, of 18,255 patients initially diagnosed in the period 2004-2005, 10,539 (57.73\%) had one tumor, 3,157 (17.29\%) had two tumors, $1,437(7.87 \%)$ had three tumors, and 3,122 (17.10\%) had four or more tumors. Overall survival (OS) was inversely related to both the maximum tumor size in 24,410 patients and to the number of tumors in 24,233 patients who underwent resection from the period 1994 to 2005. In addition, of 18,619 patients surveyed in the period 2004-2005, only 904 (4.86\%) had evidence of extrahepatic spread at the time of diagnosis. Of 17,804 patients surveyed in the same period, 13,074 (73.4\%) had serum alpha-fetoprotein (AFP) levels $<200 \mathrm{ng} / \mathrm{ml}$ and $14,074(79.0 \%)$ had serum AFP levels $<400 \mathrm{ng} / \mathrm{ml}$, respectively.

Methods of treatment for HCC can be potentially curative or palliative. Potentially curative treatments include resection, local ablation, and liver transplantation. Palliative treatments include transarterial chemoembolization (TACE), hepatic arterial infusion chemotherapy (HAIC), and systemic chemotherapy, each of which has advantages and disadvantages [9-14]. More recently, the multiple tyrosine kinase inhibitor, sorafenib, has shown efficacy in patients with HCC $[15,16]$. Response criteria for patients with HCC have been recently redefined $[17,18]$. 
Although the two-yearly surveys by the LCSGJ have assessed the incidence of HCC by demographic and clinical characteristics in addition to the survival rates of these patients, these "snapshot" surveys are only able to assess these parameters at specific points in time. It is important to determine the effectiveness of surveillance and treatment methods longitudinally. We therefore analyzed the improvements in OS in 173,378 patients who were prospectively entered into the LCSGJ registry between 1978 and 2005 and assorted into 5-year intervals by date of diagnosis. We also analyzed improvements in OS over time in patients who underwent resection, local ablation, TACE, and HAIC.

\section{Patients and Methods}

The LCSGJ Surveillance System

The LCSGJ surveillance system registers all patients in Japan diagnosed with HCC. According to the Japanese HCC practice guidelines, high risk patients are those with chronic hepatitis B and/or C virus infection or liver cirrhosis. In addition, very high risk patients are those with hepatitis B or C virus associated cirrhosis $[19,20]$.

Follow-up methods for Japanese patients at high risk for HCC has included ultrasonography of the liver and measurements of the serum concentrations of tumor markers including AFP, the lectin-binding fraction of AFP (AFP-L3), and prothrombin induced in the absence of vitamin K (PIVKA-II), also known as des- $\gamma$-carboxy-prothrombin (DCP), every six months. In very high risk patients, follow-up has consisted of ultrasonography and measurements of AFP, PIVKA-II, and/or AFP-L3 every 3-4 months. At the discretion of their physicians, these patients have also undergone dynamic computed tomography (CT)/magnetic resonance imaging (MRI) every 6-12 months [19, 20].

The LCSGJ HCC Registry

The LCSGJ registry consists of all patients diagnosed with HCC at more than 700 institutions and who were prospectively registered between 1978 and 2005, with data taken from the fifth through the eighteenth follow-up survey [7]. A total of 173,378 patients with HCC were prospectively registered between 1978 and 2005. Improvements in OS over time were assessed by dividing patients into 5-year groups. We also assessed improvements in OS in patients who underwent resection, local ablation, TACE, and HAIC over the same time periods.

The maximum tumor size at the time of diagnosis and the number of tumors were measured by ultrasonography. The tumor stage at diagnosis was defined, and AFP, PIVKA-II, and AFP-L3 concentrations were measured, as described [17].

\section{Statistical Methods}

OS was calculated by the Kaplan-Meier method, with differences between groups determined by the log rank test. Differences among three or more groups were also determined by same latter method.

\section{Results}

\section{OS over Time}

A total of 173,378 patients with HCC were prospectively registered into the LCSGJ HCC registry between 1978 and 2005. The 5- and 10-year OS rates of this cohort were $37.9 \%$ and $16.5 \%$, respectively. The mean maximum tumor size was found to decrease over time (table 1). In addition, when 5-year OS rates were assessed in patients assorted by the year of diagnosis into 5-year intervals, these rates increased over time, from $3.7 \%$ in the $1978-1980$ period to $42.7 \%$ in the 2001-2005 period, with the difference between each period and the preceding period being statistically significant $(\mathrm{p}<0.0001$ each; fig. $1 \mathrm{a})$. In addition, the median survival time in patients divided by 5 -year intervals also increased over time, from three months in the 
Table 1. Maximum tumor size at the time of initial detection in patients with HCC assorted by year of diagnosis

\begin{tabular}{llllll}
\hline \multicolumn{7}{l}{ Mean maximum tumor size $(\mathrm{cm})$} \\
Year & $\leq 2.0$ & $2.1-3.0$ & $3.1-5.0$ & $5.1-10.0$ & $\geq 10.1$ \\
\hline $1978-1980$ & $1.3 \%$ & $4.2 \%$ & $3.2 \%$ & $26.3 \%$ & $65.1 \%$ \\
$1981-1985$ & $5.3 \%$ & $18.0 \%$ & $15.6 \%$ & $28.8 \%$ & $32.3 \%$ \\
$1986-1990$ & $14.5 \%$ & $25.8 \%$ & $21.5 \%$ & $21.6 \%$ & $16.5 \%$ \\
$1991-1995$ & $20.8 \%$ & $23.3 \%$ & $25.0 \%$ & $17.2 \%$ & $13.7 \%$ \\
$1996-2000$ & $17.3 \%$ & $24.5 \%$ & $23.6 \%$ & $19.6 \%$ & $15.0 \%$ \\
$2001-2005$ & $31.2 \%$ & $24.5 \%$ & $22.3 \%$ & $15.5 \%$ & $6.5 \%$ \\
\hline
\end{tabular}

Percentages are shown of patients with HCC in each time period assorted by maximum tumor size at the time of diagnosis.
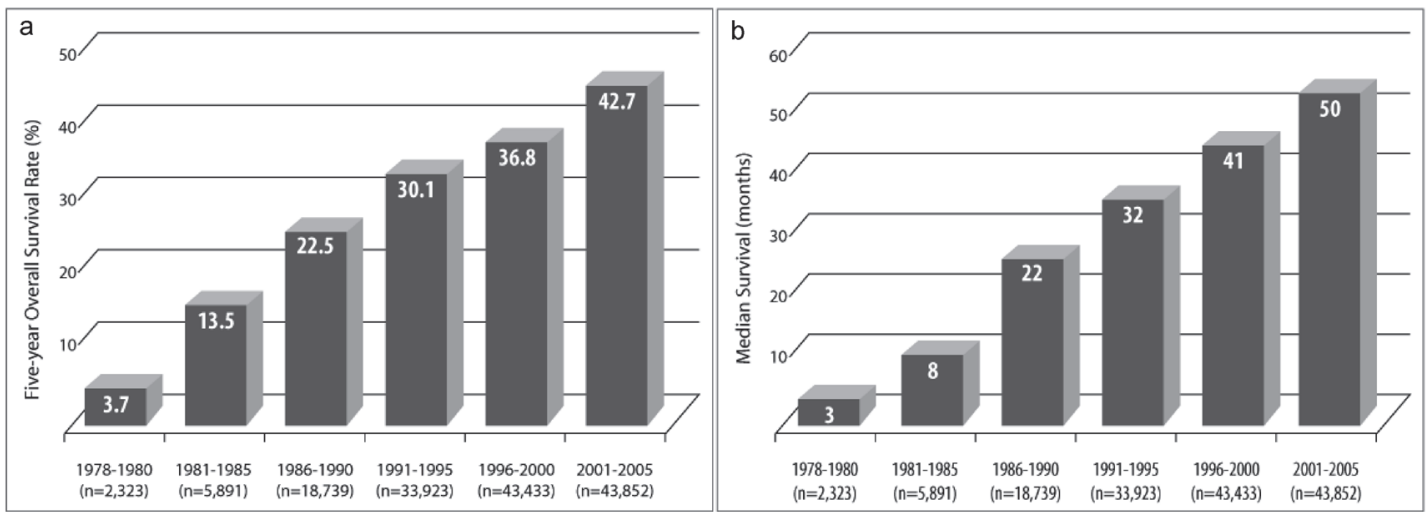

Fig. 1. Five-year OS rates (a) and (b) median OS over 5-year intervals in patients with HCC. The differences between each period and the preceding period were statistically significant $(\mathrm{p}<0.0001$ each).

1978-1980 period to 50 months in the 2001-2005 period, with the difference between each period and the preceding period also being statistically significant ( $p<0.0001$ each; fig. $1 \mathrm{~b}$ ).

\section{OS as a Function of Treatment}

To assess whether changes in OS over time were a function of initial treatment, patients were divided into those undergoing resection, local ablation, TACE and HAIC and further subdivided into 5-year intervals by year of their diagnosis with HCC. We found that the 5-year OS rates in 42,713 patients who underwent resection increased over time, from $14.5 \%$ in the 1978-1980 period to $58.4 \%$ in the $2001-2005$ period (table 2 ), as did median survival, from 13 months in the 1978-1980 period to 74 months in the 2001-2005 period (table 3). Similarly, we observed increases over time in 5-year OS rates in 37,196 patients who underwent local ablation, from $32.8 \%$ in the $1986-1990$ period to $47.6 \%$ in the $2001-2005$ period, and median survival, from 41 months in the 1986-1990 period to 59 months in the 2001-2005 period. Five-year OS rates also increased over time in 61,460 patients who underwent TACE, from $5.4 \%$ in the $1978-1980$ period to $35.0 \%$ in the $2001-2005$ period, as did median survival, from nine months in the 1978-1980 period to 42 months in the 2001-2005 period. Furthermore, 5-year OS rates increased over time, from 2.5\% in the 1978-1980 period to $31.9 \%$ in the $2001-2005$ period, in 14,246 patients who received HAIC as initial treatment, 
Table 2. Five-year OS rates (\%) in patients who underwent resection, ablation, TACE, and HAIC assorted by year of initial diagnosis

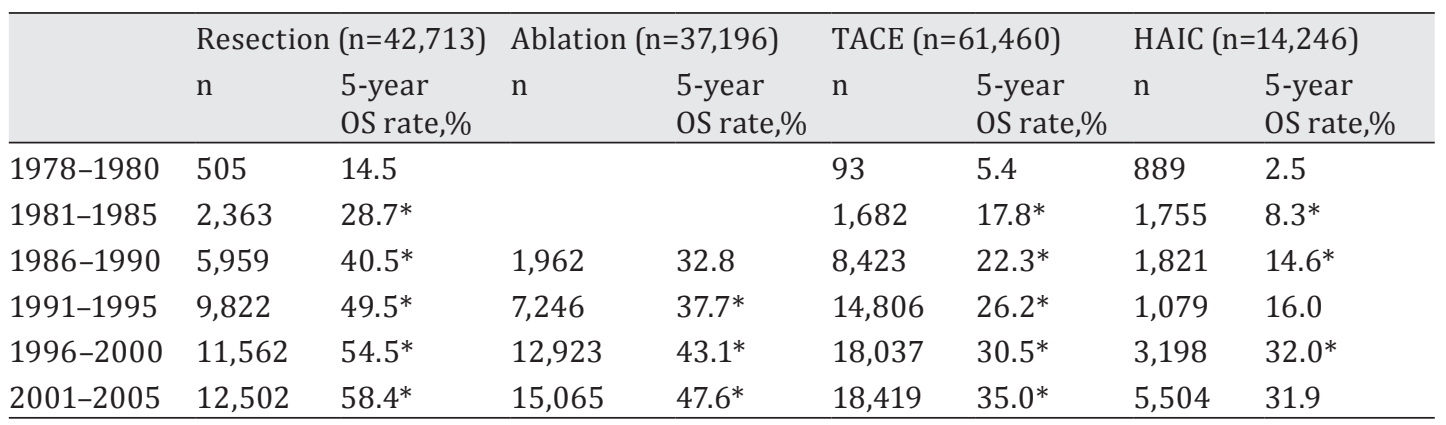

${ }^{*} \mathrm{p}<0.0001$ compared with the preceding time period.

Table 3. Median OS (months) in patients who underwent resection, ablation, TACE, and HAIC assorted by year of initial diagnosis

\begin{tabular}{|c|c|c|c|c|c|c|c|c|}
\hline & \multicolumn{2}{|c|}{ Resection $(n=42,713)$} & \multicolumn{2}{|c|}{ Ablation $(n=37,196)$} & \multicolumn{2}{|c|}{ TACE $(n=61,460)$} & \multicolumn{2}{|c|}{ HAIC (n=14,246) } \\
\hline & $\mathrm{n}$ & $\begin{array}{l}\text { Median } \\
\text { OS, months }\end{array}$ & $\mathrm{n}$ & $\begin{array}{l}\text { Median } \\
\text { OS, months }\end{array}$ & $\mathrm{n}$ & $\begin{array}{l}\text { Median } \\
\text { OS, months }\end{array}$ & n & $\begin{array}{l}\text { Median } \\
\text { OS, months }\end{array}$ \\
\hline 1978-1980 & 505 & 13 & & & 93 & 9 & 889 & 4 \\
\hline 1981-1985 & 2,363 & 28 & & & 1,682 & 17 & 1,755 & 7 \\
\hline 1986-1990 & 5,959 & 46 & 1,962 & 41 & 8,423 & 27 & 1,821 & 12 \\
\hline 1991-1995 & 9,822 & 60 & 7,246 & 47 & 14,806 & 32 & 1,079 & 10 \\
\hline $1996-2000$ & 11,562 & 69 & 12,923 & 53 & 18,037 & 37 & 3,198 & 33 \\
\hline $2001-2005$ & 12,502 & 74 & 15,065 & 59 & 18,419 & 42 & 5,504 & 31 \\
\hline
\end{tabular}

as did median survival, from four months in the 1978-1980 period to 31 months in the 20012005 period.

We also assessed 5-year OS rates over time in HCC patients with AFP levels $\geq 400 \mathrm{ng} / \mathrm{ml}$ at the time of diagnosis. We found that OS rates increased steadily over time, with the differences between each 5-year period and the preceding 5-year period being statistically significant (fig. 2).

\section{Discussion}

The results shown here, taken from the two-yearly nationwide registry maintained by the LCSGJ, clearly show that, over 28 years, the 5-year OS rates have markedly improved in patients with HCC. Although these improvements are largely due to the appropriate and intensive treatment of patients by established treatment modalities, including potentially curative treatment (resection or ablation) and mass reductive/palliative treatment (TACE or HAIC), they are also due to the establishment of the LCSGJ surveillance program throughout Japan. In addition, the establishment of additional treatment options, including molecular targeted agents such as sorafenib and their inclusion in established treatment algorithms, are expected to bring further improvements in survival in patients with HCC in Japan.

The improvements we observed in the 5-year OS rates and median OS could be correlated with the improvements in surveillance and treatment modalities over the 28-year time span. 


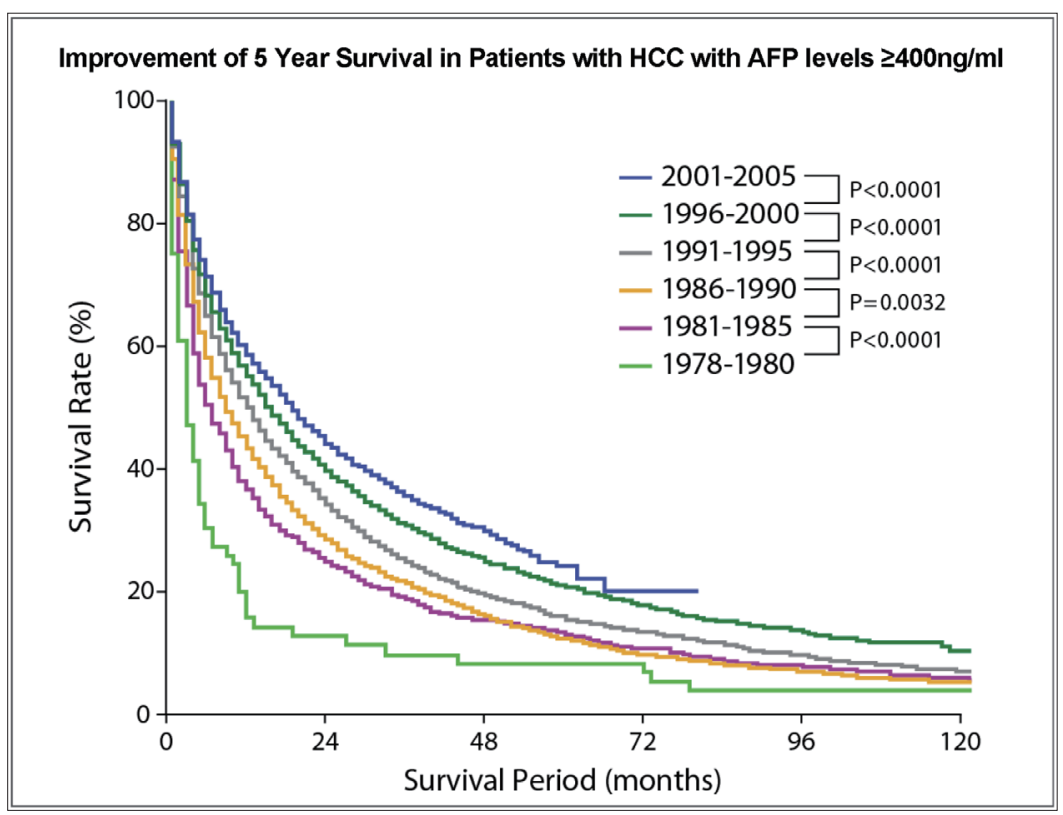

Fig. 2. Kaplan-Meier analysis of OS in patients with AFP levels $\geq 400 \mathrm{ng} / \mathrm{ml}$ at the time of diagnosis as a function of the period of diagnosis: 1978-1980 (light green), 1981-1985 (pink), 1986-1990 (yellow), 1991-1995 (gray), 1996-2000 (dark green), and 2001-2005 (blue). $\mathrm{p}<0.0001$ for all differences between each period and the preceding period, except for $\mathrm{p}<0.0032$ for 1986-1990 vs. 1981-1985.

The inclusion of ultrasound examinations and measurements of serum AFP concentration was instituted in the Japanese nationwide surveillance program in 1980. Hepatic resection and TACE were instituted as potentially curative and palliative treatments in 1985. In 1990, helical CT/MRI was introduced for diagnosis, percutaneous ethanol injection therapy was instituted in patients with HCC, and interferon treatment was instituted in patients with chronic HCV infection. In 1995, HAIC was instituted to treat HCC and measurements of DCP and AFP-L3 were instituted for patient surveillance and diagnosis. Radiofrequency ablation was instituted for curative treatment, multidetector-row CT for surveillance and diagnosis was introduced in 2000, and sorafenib was approved for the systemic treatment of patients with HCC in 2009.

\section{Comparisons with Other Countries}

Five-year OS rates for patients in the United States with liver and intrahepatic bile duct cancer have also shown slight improvements over time, from $3 \%$ for patients diagnosed in the 1975-1977 period to $11 \%$ for patients diagnosed in the 2001-2007 period [21-23]. In Korea, where guidelines recommend that individuals be monitored for HCC beginning at the age of 30 years old, the 5 -year OS rates are higher than in the US, improving from $10.7 \%$ for patients diagnosed in the 1993-1995 period to $18.9 \%$ for patients diagnosed in the 20012005 period [24]. Nevertheless, they remain lower than those in Japan, perhaps due to the longer duration of the surveillance program and its complete nationwide establishment in Japan, but which is not the case in Korea.

Over time, approximately $62 \%$ of patients with HCC in Japan have undergone potentially curative treatments (resection or ablation) as initial therapy. In comparison, only about $30 \%$ 
of patients in Western countries are eligible for potentially curative treatments at the time of their initial diagnosis [25]. The higher numbers of Japanese patients eligible for potentially curative treatment are likely to be due to the earlier detection and treatment of HCC, which is ultimately due to the establishment of comprehensive surveillance programs in patients at risk for HCC. This has undoubtedly resulted in increased 5-year OS rates in countries with surveillance programs, indicating that surveillance of patients at risk can lead to early detection and treatment [26]. The application of process-of-care quality indicators to measure evidence-practice gaps has been applied to the liver cancer registry and this may improve the quality of care in these patients [27]. Other differences between Japanese and Western populations are probably negligible in their contribution to differences in OS rates. These include differences in genetics, diet, and disease characteristics.

In conclusion, our findings show that the establishment of a nationwide HCC surveillance program in Japan has contributed to increased OS rates. These findings indicate the need for the establishment of national surveillance programs worldwide of patients at risk for HCC.

\section{Disclosure Statement}

The authors declare that they have no financial conflict of interest.

\section{References}

1 Ferlay J, Shin HR, Bray F, Forman D, Mathers C, Parkin DM: Estimates of worldwide burden of cancer in 2008: GLOBOCAN 2008. Int J Cancer 2010;127:2893-2917.

2 Asia-Pacific Working Party on Prevention of Hepatocellular Carcinoma: Prevention of hepatocellular carcinoma in the Asia-Pacific region: consensus statements. J Gastroenterol Hepatol 2010;25:657-663.

3 Song P, Tang W, Tamura S, Hasegawa K, Sugawara Y, Dong J, Kokudo N: The management of hepatocellular carcinoma in Asia: a guideline combining quantitative and qualitative evaluation. Biosci Trends 2010;4:283-287.

4 Umemura T, Ichijo T, Yoshizawa K, Tanaka E, Kiyosawa K: Epidemiology of hepatocellular carcinoma in Japan. J Gastroenterol 2009;44(Suppl 19):102-107.

5 Kudo M, Chung H, Haji S, Osaki Y, Oka H, Seki T, Kasugai H, Sasaki Y, Matsunaga T: Validation of a new prognostic staging system for hepatocellular carcinoma: the JIS score compared with the CLIP score. Hepatology 2004;40:1396-1405.

6 El-Serag HB: Hepatocellular carcinoma. N Engl J Med 2011;365:1118-1127.

7 Ikai I, Arii S, Okazaki M, Okita K, Omata M, Kojiro M, Takayasu K, Nakanuma Y, Makuuchi M, Matsuyama Y, Monden M, Kudo M: Report of the 17th Nationwide Follow-up Survey of Primary Liver Cancer in Japan. Hepatol Res 2007;37:676-691.

8 Ikai I, Kudo M, Arii S, Omata M, Kojiro M, Sakamoto M, et al: Report of the 18th Nationwide Follow-up Survey of Primary Liver Cancer in Japan. Hepatol Res 2010;40:1043-1059.

9 Takayasu K, Arii S, Ikai I, Omata M, Okita K, Ichida T, Matsuyama Y, Nakanuma Y, Kojiro M, Makuuchi M, Yamaoka Y, Liver Cancer Study Group of Japan: Prospective cohort study of transarterial chemoembolization for unresectable hepatocellular carcinoma in 8510 patients. Gastroenterology 2006;131:461-469.

10 Hasegawa K, Makuuchi M, Takayama T, Kokudo N, Arii S, Okazaki M, Okita K, Omata M, Kudo M, Kojiro M, Nakanuma Y, Takayasu K, Monden M, Matsuyama Y, Ikai I: Surgical resection vs. percutaneous ablation for hepatocellular carcinoma: a preliminary report of the Japanese nationwide survey. J Hepatol 2008;49:589594.

11 Fujita T: Hepatectomy versus radiofrequency ablation for early hepatocellular carcinoma. J Hepatol 2009;50:1051-1052, author reply 1052-1053.

12 Takayasu K, Arii S, Ikai I, Kudo M, Matsuyama Y, Kojiro M, Makuuchi M, Liver Cancer Study Group of Japan: Overall survival after transarterial lipiodol infusion chemotherapy with or without embolization for unresectable hepatocellular carcinoma: propensity score analysis. AJR Am J Roentgenol 2010;194:830-837.

13 Eguchi S, Kanematsu T, Arii S, Omata M, Kudo M, Sakamoto M, Takayasu K, Makuuchi M, Matsuyama Y, Monden M, Liver Cancer Study Group of Japan: Recurrence-free survival more than 10 years after liver resection for hepatocellular carcinoma. Br J Surg 2011;98:552-557.

14 Mazzaferro V, Regalia E, Doci R, Andreola S, Pulvirenti A, Bozzetti F, Montalto F, Ammatuna M, Morabito A, Gennari L: Liver transplantation for the treatment of small hepatocellular carcinomas in patients with cirrhosis. N Engl J Med 1996;334:693-699. 
15 Llovet JM, Ricci S, Mazzaferro V, Hilgard P, Gane E, Blanc JF, de Oliveira AC, Santoro A, Raoul JL, Forner A, Schwartz M, Porta C, Zeuzem S, Bolondi L, Greten TF, Galle PR, Seitz JF, Borbath I, Häussinger D, Giannaris T, Shan M, Moscovici M, Voliotis D, Bruix J, SHARP Investigators Study Group: Sorafenib in advanced hepatocellular carcinoma. N Engl J Med 2008;359:378-390.

16 Cheng AL, Kang YK, Chen Z, Tsao CJ, Qin S, Kim JS, Luo R, Feng J, Ye S, Yang TS, Xu J, Sun Y, Liang H, Liu J, Wang J, Tak WY, Pan H, Burock K, Zou J, Voliotis D, Guan Z: Efficacy and safety of sorafenib in patients in the Asia-Pacific region with advanced hepatocellular carcinoma: a phase III randomised, double-blind, placebo-controlled trial. Lancet Oncol 2009;10:25-34.

17 Kudo M, Ueshima K, Kubo S, Sakamoto M, Tanaka M, Ikai I, Furuse J, Murakami T, Kadoya M, Kokudo N Liver Cancer Study Group of Japan (Committee for Response Evaluation Criteria in Cancer of the Liver, Liver Cancer Study Group of Japan): Response Evaluation Criteria in Cancer of the Liver (RECICL) proposed by the Liver Cancer Study Group of Japan (2016 Revised Version). Hepatol Res 2016;46:3-9.

18 Lencioni R, Llovet JM: Modified RECIST (mRECIST) assessment for hepatocellular carcinoma. Semin Liver Dis 2010;30:52-60.

19 Makuuchi M, Kokudo N, Arii S, Futagawa S, Kaneko S, Kawasaki S, Matsuyama Y, Okazaki M, Okita K, Omata M, Saida Y, Takayama T, Yamaoka Y: Development of evidence-based clinical guidelines for the diagnosis and treatment of hepatocellular carcinoma in Japan. Hepatol Res 2008;38:37-51.

20 Kudo M, Izumi N, Kokudo N, Matsui O, Sakamoto M, Nakashima O, Kojiro M, Makuuchi M, HCC Expert Panel of Japan Society of Hepatology: Management of hepatocellular carcinoma in Japan: Consensus-Based Clinical Practice Guidelines proposed by the Japan Society of Hepatology (JSH) 2010 updated version. Dig Dis 2011;29:339-364.

21 Altekruse SF, McGlynn KA, Dickie LA, Kleiner DE: Hepatocellular carcinoma confirmation, treatment, and survival in surveillance, epidemiology, and end results registries, 1992-2008. Hepatology 2012;55:476482.

22 Ries. LAG, Melbert D, Krapcho M, et al (eds.). SEER Cancer Statistics Review, 1975-2005, National Cancer Institute, Bethesda, MD. Available at: http://seer.cancer.gov/csr/1975_2005/,2008. Accessed February $27,2012$.

23 American Cancer Society. Cancer facts and figures 2012.

24 Park JW, Korean Liver Cancer Study Group and National Cancer Center: [Practice guideline for diagnosis and treatment of hepatocellular carcinoma]. Korean J Hepatol 2004;10:88-98.

25 Llovet JM, Burroughs A, Bruix J: Hepatocellular carcinoma. Lancet 2003;362:1907-1917.

26 El-Serag HB, Davila JA: Surveillance for hepatocellular carcinoma: in whom and how? Therap Adv Gastroenterol 2011;4:5-10.

27 Higashi T, Hasegawa K, Kokudo N, Makuuchi M, Izumi N, Ichida T, Kudo M, Ku Y, Sakamoto M, Nakashima 0, Matsui O, Matsuyama Y, Sobue T, Liver Cancer Study Group of Japan: Demonstration of quality of care measurement using the Japanese liver cancer registry. Hepatol Res 2011;41:1208-1215. 